\title{
A study of admissions to paediatric beds
}

\author{
G. Rajaratnam
}

Department of Epidemiology and Community Medicine, University of Wales College of Medicine, Heath Park, Cardiff CF4 4XN, UK.

\begin{abstract}
Summary: The reasons for admission of children to paediatric beds and the appropriateness of those admissions were investigated by a retrospective study of admission notes by two paediatricians. Approximately $3 \%$ and $9 \%$ of the admissions according to assessor, were for purely social reasons and the remainder for predominantly medical reasons. There were a number of admissions for whom the assessors were unable to justify admission. Assessor 1 considered that $20 \%$ and assessor $215 \%$, of those studied could have been managed without admission. Agreement over individual cases between assessors was poor but significant. The assessors agreed that admission was appropriate in $74 \%$ and inappropriate in $8 \%$ of cases. In the remaining 18 cases, the assessors did not agree on the need for admission. It is suggested that there is a need for local specialty specific admission policies to ensure cost-effective use of facilities.
\end{abstract}

\section{Introduction}

The reasons for admission of patients to acute hospitals and the appropriateness of those admissions have not attracted much research attention in the United Kingdom. This is surprising given that inpatient care is expensive, health care resources are limited and there is unmet need (waiting list) for care.

Several specialties individually and in combination have been examined in terms of reasons for and the appropriateness of admission; paediatrics, ${ }^{1,2}$ general medicine, ${ }^{3,4}$ acute specialties apart from obstetrics ${ }^{5}$ and acute admissions irrespective of specialty. ${ }^{6}$ The results are difficult to interpret because the methodology of these studies can be criticized for one or more of the following biases: firstly, appropriateness was defined in terms of the medical model, secondly, all admissions for medical reasons were considered to be appropriate and thirdly, the results were based on the views of doctors closely involved in the care of the patients studied. In reality, the decision to admit is often made by the least experienced doctor on the firm, using the limited information available at the time of admission.

It is a difficult issue to investigate because the role of the acute hospital in terms of care provision has never been explicitly defined. Thus, there are no accepted or agreed criteria for admission. In the

Correspondence and present address: G. Rajaratnam, M.F.C.M., Department of Public Health Medicine, Bradford Health Authority, Daisy Bank, 109 Duckworth Lane, Bradford BD9 6RL, UK.

Accepted: 5 September 1990
United States, appropriateness has been defined in terms of a medical model. ${ }^{7}$ In the United Kingdomhowever, it is implicitly accepted that social circumstances are important in determining the need for acute inpatient care. ${ }^{1}$

I report a study examining the reasons for admission of children and the appropriateness of those admissions in an inner city Health District which was performed at the request of the District Health Authority, in order to inform discussions about the need for rationalization of inpatient services for children, from three separate inpatient children's units to a single inpatient unit.

\section{Methods}

A 1 in 5 random sample, stratified according to deaths and discharges from each of the three existing paediatric units in 1985 , was chosen for study. In consultation with the Division of Paediatrics, two consultant paediatricians (assessors 1 and 2) from different Health Regions were selected. Both were working in large cities and had experience of inner city paediatrics. Photocopies of admission notes only, were forwarded to the assessors who were asked to complete a questionnaire for each admission. The assessors were requested not to discuss the admissions with each other.

The following reasons for admission were listed:

(a) the diagnosis and/or severity of illness and/or complication and/or treatment

(b) social circumstances

(c) doubts about the clinical condition 
(d) education of the child and/or parents

(e) research or teaching purposes.

The assessors were asked to grade these from 4 (of major importance in determining the need for admission) to 1 (little or no importance in determining the need for admission). Two intermediate grades were used to minimize middle digit preference. An admission was for predominantly medical reasons if (a) and/or (c) were graded as 4, 3 or 2 irrespective of the grades for the others. A predominantly social admission was defined as one where (b) was graded as 4, 3 or 2 and all others as 1 .

The question on appropriateness of admission was worded in the following way, 'If, in your opinion, this patient did not require admission to an acute inpatient bed, please indicate which one of the following alternatives would be appropriate?' The assessors were asked to choose from outpatient care, day care, general practitioner care, home care (defined as cared for at home by doctors and nurses trained in paediatrics), or other.

Agreement amongst the assessors was investigated by means of Cohen's Kappa ${ }^{8}$ Kappa values greater than 0.75 represent excellent agreement, between 0.4 and 0.75 fair agreement and less than 0.4 poor agreement. If the Kappa value is significant then this would imply that agreement was greater than would be expected by chance alone. The difference between the assessors and its significance was estimated by Mcnemar's test.

\section{Results}

Of the 750 admission notes selected for study, 620 $(83 \%)$ were suitable for review by the assessors and form the basis of the analysis presented in this report. Approximately $10 \%$ were misplaced (these were not of patients who were most recently discharged) and the rest were incomplete (parts of the admission notes were missing). The numbers from each hospital were in proportion to that unit's paediatric deaths and discharges. However, there was a preponderance of patients aged 4 weeks or less in the study sample (7\%) as compared to the sampling frame $(3 \%)$. Overall, $68 \%$ of the admissions studied were referrals from casualty, $6 \%$ were referred by general practitioners, $19 \%$ were elective admissions and the remainder transfers.

\section{Reasons for admission}

In almost all cases both assessors graded reasons (c) to (e) as 1 (little or no importance in determining the need for admission). For this reason, they are excluded from the analysis that follows. Also for ease of presentation, the two intermediate grades have been combined and labelled as 'some impor- tance'. A more detailed report is available elsewhere. ${ }^{9}$

According. to both assessors the majority of admissions were for predominantly medical reasons and social circumstances were of little importance (Figures 1 and 2). Approximately $9 \%$ (assessor 1) and 3\% (assessor 2) of admissions were for predominantly social reasons. Assessors 1 and 2 were not able to justify admission in $3 \%$ and $1 \%$ of cases respectively.

\section{Need for admission}

Twenty percent and $15 \%$ of the admissions were considered, by assessors 1 and 2 respectively, to not require admission. General practitioner care was favoured by assessor 1 in $69 \%$ of these cases whereas assessor 2 chose this in $29 \%$ (Table I). The assessors agreed that admission was required in $73.5 \%$ of cases (456 patients) and not required in $8.4 \%$ (52 patients). In $18.1 \%$ ( 112 patients) cases the assessors did not agree on the need for admission.

The difference between the assessors was significant (Mcnemar's test $=3.3, P<0.01,95 \%$ confidence interval for the difference was 0.03 to 0.09 ). The agreement amongst those thought to require

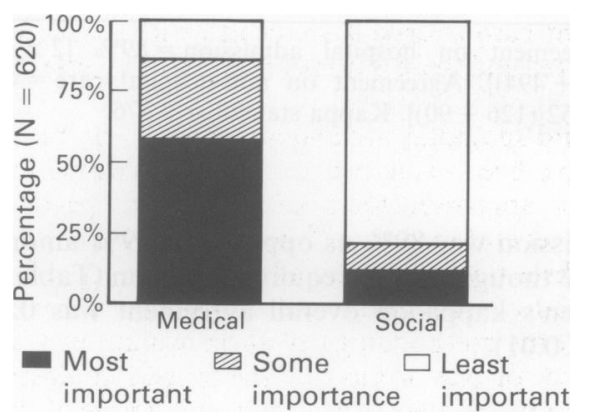

Figure 1 Importance of selected reasons for admission: Assessor 1.

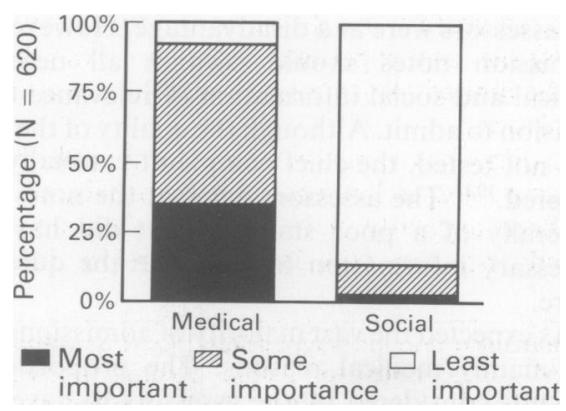

Figure 2 Importance of selected reasons for admission: Assessor 2. 
Table I Alternative patterns for care for children admitted

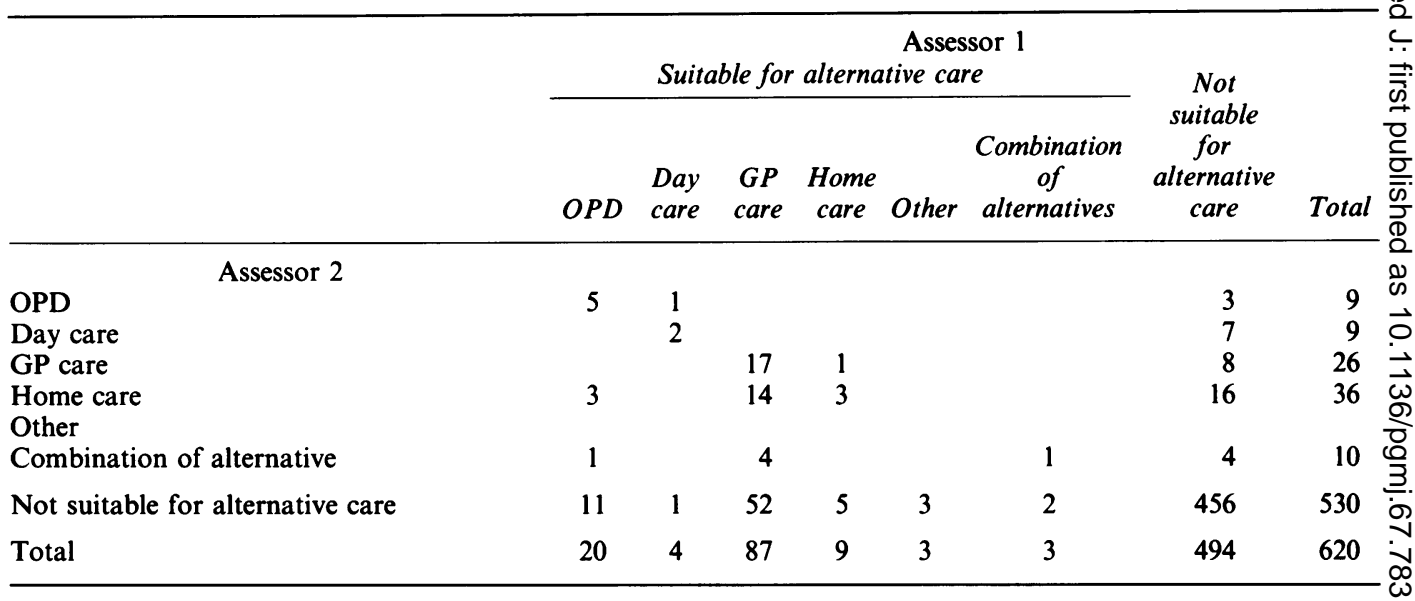

Table II Comparison of the assessors in terms of hospital and non-hospital

\begin{tabular}{lccr}
\hline & Hospital & $\begin{array}{c}\text { Assessor 1 } \\
\text { Non-hospital }\end{array}$ & Total \\
\hline \multicolumn{1}{c}{ Assessor 2 } & & & \\
Hospital & 456 & 74 & 530 \\
Non-hospital & 38 & 52 & 90 \\
Total & 494 & 126 & 620 \\
\hline
\end{tabular}

Agreeement on hospital admission $=89 \%[2 \times 456 /$ $(530+494)]$. Agreement on non-hospital care $=48 \%$ $[2 \times 52 /(126+90)]$. Kappa statistic $=0.376$.

admission was $89 \%$ as opposed to $49 \%$ amongst those thought not to require admission (Table II). Cohen's kappa on overall agreement was 0.376 $(P<0.01)$.

\section{Discussion}

The decision to admit is made in an unique environment which cannot be replicated. Clearly the assessors were at a disadvantage. However, the admission notes should contain all necessary clinical and social information of relevance to the decision to admit. Although the quality of the notes was not tested, the chief complaint is usually well covered. $^{10,11}$ The assessors felt that the notes were generally of a poor standard but did have the necessary information to complete the questionnaire.

As expected the vast majority of admissions were for mainly medical reasons. The proportion of patients considered by the assessors to have been admitted for mainly social reasons was much less than the $20 \%$ estimated by Wynn and Hull. ${ }^{2}$ This is probably due to differences in the assumptions underlying the two studies. Wynn and Hull assum- $\frac{}{I}$ ed that if the admission was not justified on medical $\vec{C}$ grounds then it was a social admission whereas in our case, for an admission to be classified as social, there had to be a positive social reason for admission in addition to lack of medical grounds for that $\vec{\theta}$ admission. Indeed, for a proportion of patients the assessors could not justify admission based on the information available within the admission notes Further, Wynn and Hull categorized patients admitted for both social and medical reasons as social admissions whereas we have categorized them as admitted for medical reasons.

The study by Wynn and Hull and the one $\overrightarrow{\vec{O}}$ reported here both involved paediatric units located within inner cities. The migratory patterns of the past century and the current demography of England and Wales, suggest that although rural areas can be as deprived as inner city areas, the particular components of deprivation are different (for example, homelessness appears to be a particular problem of inner city areas rather than rural areas). It is therefore difficult to extrapolate with any confidence from these results to what one might expect in rural areas.

In terms of appropriateness of admissions, there does not appear to be a comparable study performed in the United Kingdom. Comparisons with studies from the United States are difficult for the reason that the social dimension is excluded in the United $\mathrm{C}$ States. ${ }^{1,3}$ Our results were that approximately $20 \%$ 웅 and $15 \%$, according to assessor 1 and 2 respectively, were considered not to require inpatient care. These probably include patients whose admis- ? sion could not be justified by the assessors as well as those who had medical reasons for admission but where the assessors considered that admission was not the most appropriate mode of care. The latter group may have consisted of patients admitted 
because admission was specifically requested by the general practitioner as well as those whose admission was due to the inexperience of the admitting doctor. The latter issue is important because the general policy in acute hospitals is to encourage junior doctors to admit if at all worried. This would result in larger numbers of inappropriate admissions than if the policy stated that there must be a positive reason for every admission.

The kappa value of 0.376 shows that, although the agreement between assessors was poor, it was still greater than would be expected by chance alone. But the fact that the difference between the two assessors was significant suggests that assessor 2 was more likely to hospitalize patients than assessor 1 . This was surprising as both clinicians were working in large cities and had experience of working in inner city children's units. The difference may be due to either the assessors having different criteria for admission or, although the same criteria were used, they were weighted differently. The data presented here do not help to differentiate between these two explanations. Circumstantial evidence based on interpretation of hypothetical cases by clinicians ${ }^{12-14}$ and the unsuccessful attempt to come to a consensus view between clinicians on the appropriate use of beds ${ }^{15}$ suggest that not only do doctors use different criteria but that even when there are common criteria, they are weighted differently.

The observed difference between the two assessors may reflect two extremes or it may be consistent with variation amongst consultants in general. It is suggested that such variations ${ }^{12-16}$ stem from unresolved medical care controversies and that this affects all specialties. ${ }^{17}$ One way of reducing such differences is by using consensus development. However, Rosser's work ${ }^{15}$ demonstrating persistent differences between consultants on the appropriate use of beds despite several attempts at consensus development using the delphi method, suggest that if discussion between the two assessors had been allowed, there would still have been differences but less than that observed.

More than $90 \%$ of admissions to paediatric wards in this inner city district in 1985 were considered by 2 paediatric assessors to have been due to predominantly medical reasons. Less than $10 \%$ were admitted for predominantly social reasons. The two assessors agreed independently that $73.5 \%$ of cases were correctly admitted and that $8 \%$ should have not been admitted. They did not agree amongst the remainder and it is suggested that in these cases, admission may be doctordependent and may not necessarily be dependent upon morbidity.

In view of the increasing trend in paediatric admission, the opportunity costs of the observed variation are likely to be substantial. Further, unnecessary admission of children is considered to be harmful to children and their carers. ${ }^{19,20}$ One paediatric unit found that as a result of setting up a system of consultant screening of a proportion of admissions in addition to a follow up policy, that they only needed two of the three children's wards. ${ }^{20}$ The finding that consultants differ significantly in interpretation of the need for admission suggests that further improvements should be possible.

We would suggest that the issue of why patients are admitted and whether the admission was necessary should be regularly reviewed within the medical audit forum in all districts. In this way it should be possible to develop local, specialtyspecific guidelines and/or standards agreed by all clinicians in that specialty and locality. Nationally produced guidelines very rarely produce change in practice, ${ }^{21}$ whereas locally agreed guidelines do result in change. ${ }^{22}$ It is therefore important that whatever guidelines are produced are seen to be locally owned, take into account local circumstances and are acceptable to hospital clinicians, general practitioners and parents.

\section{Acknowledgements}

I am grateful to Dr Deirdre Cunningham and the Paediatric Division for their advice and support. I would also like to thank the two paediatric assessors without whom the study would not have been possible.

\section{References}

1. Siu, A.L., Sonnenberg, F.A., Manning, W.G. et al. Inappropriate use of hospitals in a randomized trial of health insurance plans. $N$ Engl J Med 1986, 315: 1259-1266.

2. Wynne, J. \& Hull, D. Why are children admitted to hospital? Br Med J 1977, 2: 1140-1142.

3. Kemper, K.J. Medically inappropriate hospital use in a paediatric population. $N$ Engl J Med 1988, 318: 1033-1037.

4. Crombie, D.L. \& Cross, K.W. Serious illness in hospital and at home. The Medical Press 1959, 242: 316-322 and 340-343.

5. Forsyth, G. \& Logan, R.F.L. The Demand for Medical Care: A study of the case load in the Burrow and Furness group of hospitals. Oxford University Press, London, 1960.
6. Mackintosh, J.M., McKeown, T. \& Garratt, F.N. An examination of the need for hospital admission. Lancet 1961, i: $815-818$.

7. Mushlin, A.I. \& Appel, F. Extramedical factors in the decision to hospitalize medical patients. Am J Public Health 1976, 66: 170-172.

8. Fleiss, J.L. The measurement of interrator agreement. In: Statistical Methods for Rates and Proportions, 2nd edition. John Wiley and Sons, New York, USA, 1981, chapter 13, pp. 212-236. 
9. Rajaratnam, G. MFCM Part II thesis. Faculty of Public Health Medicine of the Royal Colleges of Physicians of the United Kingdom 1989.

10. Romm, F.J. \& Puttnam, S.M. The validity of the medical record. Med Care 1981, xix: 310-313.

11. Swansea Physicians Audit Group. Audit of the quality of medical records in a district general hospital medical unit. $J \boldsymbol{R}$ Coll Physicians Lond 1983, 17: 208-212.

12. Rosenblatt, R.A. \& Moscovice, I.S. The physician as gatekeeper. Determinants of physicians' hospitalisation rates. Med Care 1984, 22: 150-159.

13. Kuder, J.M., Vilmain, J.A. \& Demlo, L.K. Exploring physician responses to patients extramedical characteristics. The decision to hospitalize. Med Care 1987, 25: 882-892.

14. Hemenway, D. \& Fallon, D. Testing for physician induced demand with hypothetical cases. Med Care 1985, 23: 344-349.

15. Rosser, R.M. The reliability and application of clinical judgement in evaluating the use of hospital beds. Med Care 1976, 14: 39-47.
16. Ham, C. Research report 2. Health care variations: assessing 3 the evidence. The King's Fund Institute, London, 1988.

17. Wennberg, J.E., Freeman, J.L. \& Culp, W.J. Are Hospitalc services rationed in New Haven or over-utilised in Boston? Lancet 1987, ii: $1185-1189$.

18. Hill, A.M. Trends in paediatric medical admissions. Br Med 1989, 298: 1479-1482. 19. Forfar, J.O. Trends in paediatric medical admissions. Br Med $\frac{\overline{\bar{\sigma}}}{\bar{\omega}}$
$J$ 1989, 298: 1711 .

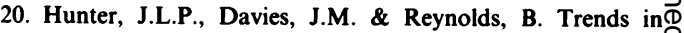
paediatric medical admissions. Br Med J 1989, 299: 516.

21. Lomas, J., Anderson, G.M., Domnick-Pierre, K. et al. Doळ̂ practice guidelines guide practice? The effect of a consensus statement on the practice of physicians. N Engl J Med 1989, 321: $1306-1311$.

22. Hall, R., Roberts, C.J., Coles, G.A. et al. The impact of guidelines in clinical outpatient practice. $J \boldsymbol{R}$ Coll Physicans Lond 1988, 22: 244-247. 